\title{
MULHERES COM RISCO CARDIOVASCULAR: REVISÃO DAS PESQUISAS DAS PÓS-GRADUAÇÕES BRASILEIRAS
}

\author{
Women with cardiovascular risk: review of research from Brazilian graduate programs \\ Mujeres con riesgo cardiovascular: revisión de las investigaciones de posgrados brasileños
}

\author{
Gabriela Oliveira \\ Universidade Federal de Santa Maria - UFSM - Santa Maria (RS) - Brasil \\ Maria Denise Schimith \\ Universidade Federal de Santa Maria - UFSM - Santa Maria (RS) - Brasil \\ Lúcia Beatriz Ressel \\ Universidade Federal de Santa Maria - UFSM - Santa Maria (RS) - Brasil
}

Lisie Alende Prates

Universidade Federal de Santa Maria - UFSM - Santa Maria (RS) - Brasil

Oclaris Lopes Munhoz

Universidade Federal de Santa Maria - UFSM - Santa Maria (RS) - Brasil

Thayná da Silva Champe

Universidade Federal de Santa Maria - UFSM - Santa Maria (RS) - Brasil

\section{RESUMO}

Objetivo: Identificar os fatores de risco cardiovasculares em mulheres, estudados em dissertações e teses defendidas em programas de pós-graduação brasileiros na área da saúde. Métodos: Revisão crítica e descritiva, realizada em maio de 2017, no banco de teses e dissertações da Coordenação de Aperfeiçoamento de Pessoal de Nível Superior (CAPES). Como estratégia de busca utilizaram-se as palavras-chave "fatores de risco cardiovascular" e "mulheres", sem restrição de área do conhecimento. A utilização dessas palavras-chave ocorreu como estratégia para alcançar o maior número de produções sobre o objeto de estudo. Foram incluídos estudos nacionais, oriundos de programas de pós-graduação e que respondiam à questão de pesquisa; e excluídos aqueles com resumos incompletos ou que estivessem indisponíveis. Não se determinou recorte temporal. Dos estudos encontrados, 23 foram selecionados para análise. Resultados: Os principais fatores de risco foram: a obesidade, o sedentarismo, a hipertensão arterial sistêmica e o desenvolvimento de pré-eclâmpsia durante a gestação, incluindo também os aspectos hormonais, como a menopausa. As pesquisas evidenciaram a importância e a necessidade de ações de prevenção e minimização dos fatores de risco, além do desenvolvimento de estratégias específicas, como o consumo da vitamina A, farinha de berinjela e chá-verde. Conclusão: Os fatores de risco prevalentes em mulheres estão relacionados às alterações metabólicas, às condições multifatoriais, a hábitos inadequados e a ciclos de vida, como a gestação e os aspectos hormonais, sugerindo a necessidade dos profissionais da saúde desenvolveram ações de prevenção e controle desses fatores.

Descritores: Fatores de risco; Doenças Cardiovasculares; Saúde da Mulher.

\section{ABSTRACT}

Objective: To identify the cardiovascular risk factors in women, studied in dissertations and theses defended in Brazilian graduate programs in the area of health sciences. Methods: Critical and descriptive review, carried out in May 2017, in the theses and dissertations repository of the Coordination for the Improvement of Higher Education Personnel (CAPES). As a search strategy, the keywords "cardiovascular risk factors" and "women" were used, without restriction on areas of knowledge. These keywords were used as a strategy to retrieve the largest number of publications on the object of study. National studies which addressed the research question and originated from graduate programs were included, and those with incomplete summaries or that were unavailable were excluded. No time limit was applied. Of the studies found, 23 were selected for analysis. Results: The main risk factors were: obesity, sedentary lifestyle, systemic arterial hypertension and the development of preeclampsia during pregnancy, also including hormonal aspects such as menopause. The studies have evidenced the importance of and the need for actions aimed at preventing and minimizing the risk factors, in addition to the development of specific strategies such as the consumption of vitamin A, eggplant flour and green tea. Conclusion: The risk factors prevalent among women are related to metabolic 
changes, multifactorial conditions, inadequate habits, and to life cycles such as gestation and the hormonal aspects, which suggests that health professionals need to develop preventive and control actions directed at such factors.

Descriptors: Risk Factors; Cardiovascular Diseases; Women's Health.

\section{RESUMEN}

Objetivo: Identificar los factores de riesgo cardiovasculares de mujeres los cuales han sido investigados en los trabajos de fin de master y tesis doctorales leidas en los programas de posgrado brasileños de la área de la salud. Métodos: Revisión crítica y descriptiva realizada en mayo de 2017 con el banco de tesis doctorales y trabajos de fin de master de la Coordinación de Perfeccionamiento de Personal de Nivel Superior (CAPES). Se utilizaron las palabras-clave "factores de riesgo cardiovascular" y "mujeres" como la estrategia de búsqueda sin restricción del área del conocimiento. La utilización de esas palabras-clave se dio como estrategia para alcanzar el mayor número de publicaciones sobre el objeto de estudio. Se incluyeron los estudios nacionales de programas de posgrado y que contestaban la pregunta de la investigación y se excluyeron aquellos con resúmenes incompletos o que no estuvieran disponibles. No se ha definido el recorte del tiempo. De los estudios encontrados, 23 han sido elegidos para el análisis. Resultados: Los principales factores de riesgo fueron la obesidad, el sedentarismo, la hipertensión arterial sistémica y el desarrollo de preeclampsia durante el embarazo incluyendo también los aspectos hormonales como la menopausia. Las investigaciones han evidenciado la importancia y la necesidad de acciones de prevención y disminución de los factores de riesgo además del desarrollo de estrategias especificas como el consumo de la vitamina A, la harina de berenjena y el té verde. Conclusión: Los factores de riesgo prevalentes en las mujeres se relacionan con las alteraciones metabólicas, las condiciones multifactoriales, los hábitos inadecuados y los ciclos de la vida como el embarazo y los aspectos hormonales lo que sugiere la necesidad del desarrollo de acciones de prevención y control de eses factores de parte de los profesionales sanitarios.

Descriptores: Factores de Riesgo; Enfermedades Cardiovasculares; Salud de la Mujer.

\section{INTRODUÇÃO}

Tem sido observada alteração no perfil da mortalidade da população, com aumento no número de óbitos por doenças crônicas não transmissíveis $(\mathrm{DCNT})^{(1)}$. No Brasil, as DCNT atingiram 72,7\% dos óbitos em 2011, com destaque para as doenças do aparelho circulatório (30,4\%), as neoplasias $(16,4 \%)$, a diabetes mellitus (DM) $(5,3 \%)$ e doenças respiratórias $(6,0 \%)^{(2)}$. Dentre as DCNT, as doenças cardiovasculares (DCV) afetam o sistema circulatório, causando distúrbios no coração e nos vasos sanguíneos ${ }^{(3)}$.

Entre as implicações para o desenvolvimento dessas patologias estão os fatores de risco ${ }^{(4)}$, que podem ser divididos em modificáveis (ou controláveis) e não modificáveis. Os modificáveis envolvem aspectos comportamentais e ambientais, como colesterol sérico elevado, hipertensão arterial sistêmica (HAS), tabagismo, inatividade física, DM, obesidade, estresse, obesidade e uso de anticoncepcional. Os fatores de risco não modificáveis têm etiologia biológica ou genética, abrangendo a hereditariedade, sexo e idade avançada ${ }^{(5)}$.

Na população feminina, a elevada prevalência de fatores de risco para DCV pode estar associada à inserção da mulher no mercado de trabalho, que a expõe ao estresse, ao sedentarismo, ao alcoolismo, ao tabagismo e a hábitos alimentares inadequados ${ }^{(6)}$. Ainda destaca-se a sobrecarga de responsabilidades com o trabalho, os cuidados com a família e as atividades domésticas atreladas entre elas ${ }^{(7)}$.

Estudo epidemiológico apresentou associação significativa entre o uso de contraceptivos orais e o aumento de risco para o desenvolvimento das $\mathrm{DCV}^{(8)}$. Doenças como DM e HAS também prevalecem entre as mulheres ${ }^{(9)}$.

Assim, compreende-se a importância de estudos envolvendo os fatores de risco para DCV na população feminina. A justificativa deste estudo guarda relação com as mudanças de hábitos na população, a crescente incidência de fatores de risco em mulheres, a elevada prevalência da mortalidade por DCV, a gravidade de suas complicações e o alto custo para o sistema de saúde.

Ademais, a temática supracitada é referenciada na Agenda Nacional de Prioridades em Pesquisa em Saúde, que incentiva a realização de pesquisas que visam contribuições na prevenção das doenças cardiovasculares e na qualidade de vida da população. Entende-se que, ao conhecer os fatores de risco cardiovasculares em mulheres no Brasil, é possível direcionar ações e planejamento de estratégias para a promoção à saúde e, assim, contribuir na prevenção e controle das DCV. Além disso, será possível apontar possibilidades para futuras pesquisas, contribuindo para o ensino e a prática profissional.

O estudo teve como questão de pesquisa: quais os fatores de risco cardiovasculares em mulheres que estão sendo pesquisados pelos programas de pós-graduação no Brasil? Com o intuito de responder à questão de pesquisa, elencou-se como objetivo identificar os fatores de risco cardiovasculares em mulheres, estudados em dissertações e teses defendidas nos programas de pós-graduação brasileiros na área da saúde.

\section{MÉTODOS}

Trata-se de uma revisão do tipo crítica e descritiva, com foco nas dissertações e teses defendidas nos programas de 
pós-graduação na área da saúde no Brasil ${ }^{(10)}$. A busca foi desenvolvida no banco de teses e dissertações da Coordenação de Aperfeiçoamento de Pessoal de Nível Superior (CAPES), em maio de 2017.

Como estratégia de busca, utilizaram-se as palavras-chave "fatores de risco cardiovascular" e "mulheres", sem restrição de área do conhecimento. A utilização dessas palavras-chave ocorreu como uma estratégia para alcançar o maior número de produções. Não houve determinação de recorte temporal.

Utilizaram-se como critérios de inclusão pesquisas nacionais, oriundas de programas de pós-graduação, que respondiam à questão de pesquisa. Os critérios de exclusão compreenderam os estudos com resumos incompletos ou que estavam indisponíveis. Não foram encontrados estudos duplicados.

Encontraram-se 652 produções; sendo selecionadas, após leitura de títulos, 55 pesquisas. Após a aplicação dos critérios de seleção, o corpus final para o presente estudo foi constituído por 23 produções (Figura 1).

\begin{tabular}{|c|}
\hline \multicolumn{1}{|c|}{ "Fatores de risco cardiovascular" } \\
\hline \multicolumn{2}{|c|}{652 Produções } \\
\hline LOCALIZADOR: mulheres \\
\hline 55 Produções \\
\hline Estudos incompletos e/ou Indisponíveis (13 excluídos) \\
\hline 42 Produções \\
\hline Não responderam à questão norteadora (19 excluídos) \\
\hline Total: 23 Produções \\
\hline
\end{tabular}

Figura 1 - Fluxograma de seleção das produções acerca dos fatores de risco cardiovascular em mulheres, indexados na Coordenação de Aperfeiçoamento de Pessoal de Nível Superior (CAPES).

Os aspectos éticos deste estudo foram preservados. Todos os autores consultados foram referenciados adequadamente, conforme a Lei de Direitos Autorais n ${ }^{\circ} .9 .610 / 98^{(11)}$.

\section{RESULTADOS}

Após a leitura dos estudos selecionados, elaborou-se um quadro sinóptico contendo os seguintes dados: autor/ano, tipo de estudo, objetivo e principais resultados. Os dados foram interpretados por abordagem qualitativa e descritiva, sendo realizada a análise crítica dos resultados. Dividiram-se os principais achados em dois eixos temáticos: caracterização dos estudos relacionados aos fatores de risco cardiovasculares em mulheres; fatores de risco cardiovasculares em mulheres e a importância da prevenção e controle.

\section{Caracterização dos estudos relacionados aos fatores de risco cardiovasculares em mulheres}

Em relação à região em que os estudos ocorreram, predominou a Sudeste com 11 produções, seguida da região Nordeste com seis estudos e as regiões Sul e Centro-Oeste com três estudos cada. Os registros do Diretório dos Grupos de Pesquisa no Brasil, referentes ao período de 1993 a 2016, apontam o predomínio dos Grupos de Pesquisa (GP) na região Sudeste, o que justifica os dados encontrados, os quais indicam o grande número de pesquisas realizadas na região supracitada ${ }^{(12)}$.

Verificou-se o predomínio de estudos no ano de 2013, o que pode estar relacionado com a publicação da I Diretriz Brasileira de Prevenção Cardiovascular, a qual apresenta os fatores de risco cardiovasculares e tem como principal proposta a uniformização das condutas para a prevenção dessas doenças ${ }^{(5)}$. Além dessa diretriz, no ano de 2010, o Ministério da Saúde (MS) lançou o Caderno de Atenção Primária de Rastreamento, que aborda a prevenção e estratificação de riscos na atenção básica, tendo como ênfase as DCV e seus fatores de risco ${ }^{(13)}$. Outra questão a ser destacada é que todos os estudos foram defendidos a partir dos anos 2000, o que se justifica pelo fato de que houve crescente incidência e prevalência dessas doenças devido, principalmente, ao envelhecimento da população e às mudanças no estilo de vida ${ }^{(14)}$.

Em relação à abordagem metodológica, prevalecem os estudos do tipo quantitativo. O predomínio do delineamento quantitativo pode estar relacionado ao fato de que essa abordagem busca levantar frequências, dados, indicadores e prevalências de determinado problema ou doenças ${ }^{(15)}$, o que justifica a sua utilização pelos pesquisadores. No que diz respeito à classificação dos estudos, 13 eram dissertações e dez, teses. 
No que se refere à área do conhecimento, a Medicina predominou, com 12 estudos. Após ela, a área da Educação Física, com seis produções; a Nutrição, com três; e a Enfermagem e a Fisioterapia, com um estudo cada. Diante disso, ressalta-se a importância de a Enfermagem, em especial, explorar e se apropriar dessa temática, considerando o importante papel que o enfermeiro pode desempenhar nas ações de educação em saúde e da sua relação com a comunidade, podendo atuar tanto na prevenção como na identificação precoce e no controle dos fatores de risco cardiovasculares na população ${ }^{(16)}$.

\section{Fatores de risco cardiovasculares em mulheres e a importância da prevenção e controle}

$\mathrm{Na}$ análise, identificaram-se pesquisas que abordaram as alterações metabólicas, como a dislipidemia ${ }^{(17,18)}$, e outro que abordou as condições multifatoriais, como a hipertensão arterial sistêmica (HAS) ${ }^{(19)}$. Alguns estudos relacionam ao comportamento e aos hábitos de vida dos indivíduos como, por exemplo, obesidade ${ }^{(18-22)}$ e o sedentarismo ${ }^{(23)}$, além daqueles que envolvem os ciclos de vida da mulher e os aspectos hormonais, como a Pré-Eclâmpsia $(\mathrm{PE})^{(24,25)}$, a Síndrome do Ovário Policístico (SOP) ${ }^{(26-30)}$, menopausa e pós-menopausa ${ }^{(31,32)}$. Uma pesquisa ${ }^{(33)}$ abordou a relação do risco cardiovascular ocasionado pela dislipidemia em mulheres com câncer de mama, evidenciando a necessidade de incluir rotineiramente a avaliação do perfil lipídico nessa população.

Um estudo apresentou relação indireta da migrânea com o risco de desenvolvimento das DCV devido ao sedentarismo, à depressão, à obesidade e a altos níveis de colesterol $^{(23)}$. Percebeu-se que algumas pesquisas refutaram a relação de fatores investigados com o risco cardiovascular, como a disfunção tiroidiana subclínica ${ }^{(34)}$ e a sarcopenia ${ }^{(35)}$.

Além dos fatores de risco descritos, os estudos evidenciaram a importância e a necessidade de ações visando a prevenção e minimização dos fatores de riscos cardiovasculares ${ }^{(27)}$. Autores ressaltam a importância de integrar estratégias para a prevenção das DCV, como o desenvolvimento de terapia interdisciplinar ${ }^{(19,28)}$, além da adoção de hábitos saudáveis, como a prática de atividade física ${ }^{(18,21,22,36)}$.

Os estudos abordaram ainda estratégias específicas para a prevenção e o controle dos fatores de risco cardiovasculares, como o consumo da farinha de berinjela $\mathrm{a}^{(37)} \mathrm{e}$ a ingesta de vitamina $\mathrm{A}^{(20)}$. Os dois estudos evidenciaram que esses componentes contribuem na capacidade antioxidante e, consequentemente, facilitam o controle de massa corporal. O consumo de proteína de soja isolada também melhora os níveis de colesterol total em mulheres adultas ${ }^{(38)}$. Por fim, um estudo ${ }^{(39)}$ destaca o benefício do chá-verde sobre a pressão arterial, contribuindo para o seu controle.

Quadro I - Quadro sinóptico das produções selecionadas acerca dos fatores de risco cardiovascular em mulheres pesquisados pelos programas de Pós-Graduação brasileiros.

\begin{tabular}{|c|c|c|c|c|}
\hline Autor & Ano & $\begin{array}{l}\text { Tipo de } \\
\text { Estudo }\end{array}$ & Objetivo & Principais Resultados \\
\hline $\operatorname{Jose}^{(17)}$ & 2016 & $\begin{array}{l}\text { Dissertação } \\
\text { Pesquisa } \\
\text { quantitativa }\end{array}$ & $\begin{array}{l}\text { Verificar a associação entre perfil } \\
\text { lipídico e fatores de risco cardiovascular } \\
\text { em uma mulheres. }\end{array}$ & $\begin{array}{l}\text { Observou-se alta prevalência de HAS, } \\
\text { hipercolesterolemia, níveis elevados de } \\
\text { triglicerídeos e níveis elevados de glicemia e os } \\
\text { fatores de risco predominantes foram o sobrepeso, } \\
\text { obesidade e sedentarismo. } \\
\text { Associação significativa entre colesterol e idade, } \\
\text { colesterol e Proteína C reativa, triglicérides e } \\
\text { risco de circunferência da cintura e triglicérides } \\
\text { e relação cintura/quadril. } \\
\text { Importância de se avaliar os níveis séricos de } \\
\text { colesterol em mulheres como preditor de eventos } \\
\text { cardiovasculares. }\end{array}$ \\
\hline Cerqueira $^{(18)}$ & 2011 & $\begin{array}{l}\text { Dissertação } \\
\text { Pesquisa } \\
\text { quantitativa }\end{array}$ & $\begin{array}{l}\text { Analisar a presença de fatores de risco } \\
\text { em mulheres praticante de ginástica. }\end{array}$ & $\begin{array}{l}\text { Elevada prevalência de fatores de risco } \\
\text { cardiovascular nas alunas participantes do projeto } \\
\text { de ginástica, consumo excessivo de ácidos graxos } \\
\text { saturados, além de perfil lipídico e glicemia de } \\
\text { jejum inadequados. }\end{array}$ \\
\hline Queiroz $^{19)}$ & 2013 & $\begin{array}{l}\text { Dissertação } \\
\text { Pesquisa } \\
\text { quantitativa }\end{array}$ & $\begin{array}{l}\text { Investigar os efeitos de uma terapia } \\
\text { interdisciplinar na composição corporal, } \\
\text { nos fatores de risco cardiovascular e } \\
\text { marcadores inflamatórios. }\end{array}$ & $\begin{array}{l}\text { Após a intervenção, houve redução significante } \\
\text { no IMC, representando uma perda de peso em } \\
\text { torno de } 5 \% \text {. } \\
\text { O consumo alimentar também diminuiu, } \\
\text { apresentando melhora da qualidade da alimentação } \\
\text { e mantendo-se dentro da \% de adequação de cada } \\
\text { macronutriente. }\end{array}$ \\
\hline
\end{tabular}




\begin{tabular}{|c|c|c|c|c|}
\hline Bento $^{(20)}$ & 2014 & $\begin{array}{c}\text { Tese } \\
\text { Pesquisa } \\
\text { quantitativa }\end{array}$ & $\begin{array}{l}\text { Investigar o estado nutricional de } \\
\text { vitamina A por meio dos indicadores } \\
\text { bioquímico e funcional e sua associação } \\
\text { com o índice de massa corpora e a } \\
\text { adiposidade corporal, DM2 e estresse } \\
\text { oxidativo em mulheres com ingestão } \\
\text { dietética recomendada de vitamina A. }\end{array}$ & $\begin{array}{l}\text { Inadequação do estado nutricional de vitamina } \\
\text { A associou-se ao excesso de peso, obesidade, } \\
\text { adiposidade corporal e ao estresse oxidativo. } \\
\text { O conhecimento do estado nutricional da vitamina } \\
\text { A pode contribuir para subsidiar novas estratégias } \\
\text { dietéticas eficazes para um melhor controle de } \\
\text { massa corporal, além de contribuir para o aumento } \\
\text { da capacidade antioxidante e a prevenção de } \\
\text { fatores de risco cardiovasculares. }\end{array}$ \\
\hline Tibana $^{(21)}$ & 2013 & $\begin{array}{l}\text { Dissertação } \\
\text { Pesquisa } \\
\text { quantitativa }\end{array}$ & $\begin{array}{l}\text { Avaliar os efeitos agudos e crônicos } \\
\text { do treinamento de força (TF) sobre } \\
\text { os fatores de risco cardiovascular em } \\
\text { mulheres com sobrepeso/obesidade e/ } \\
\text { ou síndrome metabólica. }\end{array}$ & $\begin{array}{l}\text { A sessão aguda do TF é capaz de diminuir a PA } \\
\text { clínica e ambulatorial tanto em mulheres com } \\
\text { sobrepeso/obesidade como em mulheres com } \\
\text { Síndrome Metabólica. } \\
\text { O TF realizado durante oito semanas sem o } \\
\text { controle dietético e sem a realização de exercícios } \\
\text { aeróbios foi pouco efetivo em alterar a glicose } \\
\text { sanguínea, PA, circunferência da cintura, os } \\
\text { triglicerídeos e o HDL. }\end{array}$ \\
\hline Chagas $^{(22)}$ & 2013 & $\begin{array}{l}\text { Dissertação } \\
\text { Pesquisa } \\
\text { quantitativa }\end{array}$ & $\begin{array}{l}\text { Avaliar o efeito de vinte semanas } \\
\text { de exercício aeróbio contínuo de } \\
\text { intensidade moderada combinado com } \\
\text { treinamento de força, sobre os fatores de } \\
\text { risco cardiovasculares e os marcadores } \\
\text { inflamatórios IL10, IL6 e TNF-alfa; } \\
\text { em mulheres pós-menopausa com } \\
\text { obesidade. }\end{array}$ & $\begin{array}{l}\text { A reprodução das recomendações de exercício } \\
\text { físico do Colégio Americano de Medicina do } \\
\text { Esporte, a partir de métodos indiretos para } \\
\text { avaliação, prescrição e monitoramento de } \\
\text { programas de exercício físico em Unidade de } \\
\text { Saúde da Família, mostrou-se efetiva em reduzir o } \\
\text { processo inflamatório de mulheres obesas na pós- } \\
\text { menopausa, em especial sobre as concentrações } \\
\text { de TNF-alfa; e IL6, como também do risco } \\
\text { cardiovascular. }\end{array}$ \\
\hline Rockett $^{(23)}$ & 2013 & $\begin{array}{l}\text { Dissertação } \\
\text { Pesquisa } \\
\text { quantitativa }\end{array}$ & $\begin{array}{l}\text { Investigar a presença de fatores de risco } \\
\text { cardiovascular em mulheres adultas } \\
\text { portadoras de migrânea e comparar } \\
\text { com controles sadios. }\end{array}$ & $\begin{array}{l}\text { Os resultados apontam nitidamente para } \\
\text { sedentarismo e depressão e possíveis distúrbios } \\
\text { do sono em pacientes com migrânea, além de } \\
\text { plausível sobreposição de efeito da obesidade e } \\
\text { da migrânea sobre os níveis séricos de HDL-c e } \\
\text { escore de risco de Framingham. }\end{array}$ \\
\hline Ferreira $^{(24)}$ & 2016 & $\begin{array}{c}\text { Tese } \\
\text { Pesquisa } \\
\text { quantitativa }\end{array}$ & $\begin{array}{l}\text { Avaliar a frequência de fatores de } \\
\text { risco cardiovascular em mulheres com } \\
\text { história de Pré-Eclâmpsia (PE) há } 12 \\
\text { meses e sua associação com hipertrofia } \\
\text { miocárdica e espessura médio-intimal } \\
\text { de carótidas (EMIC); avaliar o efeito } \\
\text { da hipertrofia miocárdica na função do } \\
\text { ventrículo esquerdo e na capacidade } \\
\text { funcional. }\end{array}$ & $\begin{array}{l}\text { É alta a prevalência de fatores de risco } \\
\text { cardiovascular doze meses após o evento de PE. } \\
\text { Foi observada elevada frequência de Hipertrofia } \\
\text { miocárdica, que foi associada a alterações dos } \\
\text { índices de função diastólica e ao prejuízo da } \\
\text { capacidade funcional. Dentre os fatores de risco, } \\
\text { o excesso de peso e aumento da circunferência } \\
\text { abdominal têm papel de destaque em aumentar } \\
\text { o risco de hipertrofia miocárdica } 12 \text { meses após } \\
\text { episódio de PE. }\end{array}$ \\
\hline Andrade $^{(25)}$ & 2013 & $\begin{array}{c}\text { Tese } \\
\text { Pesquisa } \\
\text { quantitativa }\end{array}$ & $\begin{array}{l}\text { Analisar a prevalência da síndrome } \\
\text { metabólica (SM) e fatores associados } \\
\text { em mulheres que apresentaram pré- } \\
\text { eclâmpsia (PE) e gestação normotensa } \\
\text { há cinco anos. }\end{array}$ & $\begin{array}{l}\text { As mulheres com PE prévia apresentaram uma } \\
\text { elevada prevalência da SM e de seus componentes } \\
\text { individuais em relação às normotensas, em } \\
\text { particular, a pressão arterial sistólica e diastólica } \\
\text { alteradas, os níveis baixos da concentração de } \\
\text { lipoproteínas de alta densidade e a hiperglicemia. }\end{array}$ \\
\hline
\end{tabular}




\begin{tabular}{|c|c|c|c|c|}
\hline $\operatorname{Costa}^{(26)}$ & 2009 & $\begin{array}{l}\text { Dissertação } \\
\text { Pesquisa } \\
\text { quantitativa }\end{array}$ & $\begin{array}{l}\text { Analisar a acurácia diagnóstica } \\
\text { da circunferência da cintura (CC), } \\
\text { relação cintura-estatura (RCEst), razão } \\
\text { cintura-quadril e índice de conicidade } \\
\text { para detecção de fatores de risco } \\
\text { cardiovascular (FRCV) e síndrome } \\
\text { metabólica (SM) em mulheres com } \\
\text { síndrome dos ovários policísticos (SOP). }\end{array}$ & $\begin{array}{l}\text { Nossos dados enfatizam a importância da } \\
\text { avaliação antropométrica no rastreamento do } \\
\text { risco cardiovascular em mulheres com SOP, } \\
\text { destacando-se a relevância da RCEst na predição } \\
\text { de FRCV clássicos e a necessidade de considerar } \\
\text { pontos de corte específicos para mulheres com } \\
\text { SOP para discriminação de SM. }\end{array}$ \\
\hline Azevedo(27) & 2010 & $\begin{array}{l}\text { Dissertação } \\
\text { Pesquisa } \\
\text { quantitativa }\end{array}$ & $\begin{array}{l}\text { Investigar a prevalência de níveis } \\
\text { pressóricos elevados em pacientes com } \\
\text { síndrome dos ovários policísticos (SOP) } \\
\text { e correlacionar os níveis de Pressão } \\
\text { Arterial (PA) com outros Fatores de } \\
\text { Risco Cardiovascular (FRC). }\end{array}$ & $\begin{array}{l}\text { O grupo com SOP apresentou prevalência de PA } \\
\text { alterada significativamente superior ao grupo } \\
\text { controle. } \\
\text { Mulheres com SOP apresentaram valores médio } \\
\text { superiores de PA sistólica, IMC, Circunferência } \\
\text { da Cintura (CC), triglicerídeos, glicemia de } \\
\text { jejum, HDL, colesterol em comparação ao grupo } \\
\text { controle. } \\
\text { Frequência de mulheres com valores acima do } \\
\text { limite da normalidade das cargas pressóricas foi } \\
\text { significativamente maior no grupo SOP em relação } \\
\text { ao grupo controle. }\end{array}$ \\
\hline Soares $^{(28)}$ & 2007 & $\begin{array}{l}\text { Tese } \\
\text { Pesquisa } \\
\text { quantitativa }\end{array}$ & $\begin{array}{l}\text { Avaliar fatores de risco cardiovascular } \\
\text { em mulheres brasileiras com síndrome } \\
\text { dos ovários policísticos (SOP), } \\
\text { através da utilização de múltiplos } \\
\text { parâmetros, incluindo a determinação } \\
\text { da prevalência de síndrome metabólica } \\
\text { e seus componentes e pesquisa de } \\
\text { microalbuminúria como marcador de } \\
\text { um possível dano renal precoce nessas } \\
\text { pacientes. }\end{array}$ & $\begin{array}{l}\text { Alta prevalência de síndrome metabólica e seus } \\
\text { componentes individuais em mulheres brasileiras } \\
\text { com SOP. } \\
\text { Elevado percentual de mulheres com níveis } \\
\text { de excreção urinária de albumina em faixas } \\
\text { significativamente associadas com aumento do } \\
\text { risco para eventos cardiovasculares. }\end{array}$ \\
\hline Soares ${ }^{(29)}$ & 2008 & $\begin{array}{l}\text { Dissertação } \\
\text { Pesquisa } \\
\text { quantitativa }\end{array}$ & $\begin{array}{l}\text { Avaliar a presença de marcadores } \\
\text { precoces de DCV em mulheres jovens } \\
\text { e não-obesas com SOP. }\end{array}$ & $\begin{array}{l}\text { Mulheres jovens com SOP apresentam alterações } \\
\text { na elasticidade vascular mesmo na ausência } \\
\text { de clássicos fatores de risco para DCV, como: } \\
\text { resistência à insulina, hipertensão ou obesidade. }\end{array}$ \\
\hline Santos ${ }^{(30)}$ & 2014 & $\begin{array}{l}\text { Tese } \\
\text { Pesquisa } \\
\text { quantitativa }\end{array}$ & $\begin{array}{l}\text { Avaliar o risco de Framingham a } \\
\text { prevalência de evento cardiovascular } \\
\text { em mulheres com SOP. }\end{array}$ & $\begin{array}{l}\text { A SOP está associada a maior risco de Framingham } \\
\text { alto, em mulheres em idade reprodutiva. No } \\
\text { entanto, essa associação não correspondeu à maior } \\
\text { ocorrência de eventos cardiovasculares, o que foi } \\
\text { atribuído à baixa faixa etária desta população. }\end{array}$ \\
\hline Colpani ${ }^{(31)}$ & 2015 & $\begin{array}{l}\text { Tese } \\
\text { Pesquisa } \\
\text { quantitativa }\end{array}$ & $\begin{array}{l}\text { Comparar dois instrumentos de } \\
\text { avaliação de nível de atividade física, } \\
\text { Questionário Internacional de Atividade } \\
\text { Física-versão curta (IPAQ-SF) e } \\
\text { pedômetro em mulheres na menopausa; } \\
\text { verificar o nível de atividade física } \\
\text { através do pedômetro e seu efeito em } \\
\text { fatores de risco para DCV em mulheres } \\
\text { na menopausa; analisar os fatores de } \\
\text { risco para mortalidade nesta coorte de } \\
\text { mulheres. }\end{array}$ & $\begin{array}{l}\text { A maior causa de mortalidade foi por DCV. } \\
\text { A diabetes mellitus (DM) e a obesidade central } \\
\text { foram associados a maior mortalidade total. } \\
\text { Fatores de risco tradicionais como sedentarismo, } \\
\text { maior consumo de bebida alcoólica e obesidade } \\
\text { estão diretamente associadas a um pior perfil } \\
\text { cardiovascular em mulheres na menopausa. } \\
\text { O DM em mulheres na menopausa está associado } \\
\text { a um maior risco de mortalidade total. }\end{array}$ \\
\hline
\end{tabular}




\begin{tabular}{|c|c|c|c|c|}
\hline Carvalho ${ }^{(32)}$ & 2013 & $\begin{array}{l}\text { Dissertação } \\
\text { Pesquisa } \\
\text { quantitativa }\end{array}$ & $\begin{array}{l}\text { Avaliar o consumo alimentar e a } \\
\text { prevalência de síndrome metabólica } \\
\text { (SM) em mulheres pós-menopausa, de } \\
\text { acordo com o nível de atividade física. }\end{array}$ & $\begin{array}{l}\text { As mulheres ativas na pós-menopausa tiveram } \\
\text { maior consumo diário de alimentos protetores para } \\
\text { doenças cardiovasculares (DCV), assim como os } \\
\text { alimentos de risco para tais doenças foram mais } \\
\text { consumidos pelas mulheres inativas. } \\
\text { A prevalência de SM nas mulheres inativas foi } \\
\text { maior do que nas mulheres ativas. }\end{array}$ \\
\hline Sousa $\mathrm{EP}^{(33)}$ & 2013 & $\begin{array}{l}\text { Dissertação } \\
\text { Pesquisa } \\
\text { quantitativa }\end{array}$ & $\begin{array}{l}\text { Avaliar o risco de doença cardiovascular } \\
\text { em mulheres de meia idade com câncer } \\
\text { de mama. }\end{array}$ & $\begin{array}{l}\text { A prevalência de fatores de risco cardiovascular } \\
\text { foi elevada em mulheres de meia-idade com câncer } \\
\text { de mama e a maior parte dessas apresentou risco } \\
\text { cardiovascular moderado e alto. } \\
\text { Necessidade de incluir na rotina de seguimento } \\
\text { de mulheres com câncer de mama a avaliação do } \\
\text { perfil lipídico e do risco de DCV, atentando-se para } \\
\text { o adequado controle dos níveis séricos de lipídios. }\end{array}$ \\
\hline Olmos ${ }^{(34)}$ & 2007 & $\begin{array}{l}\text { Tese } \\
\text { Pesquisa } \\
\text { quantitativa }\end{array}$ & $\begin{array}{l}\text { Determinar a frequência das disfunções } \\
\text { tireoidianas subclínicas e sua associação } \\
\text { com fatores de risco cardiovasculares } \\
\text { tradicionais e com alguns fatores } \\
\text { psicossociais em mulheres com } 40 \text { anos } \\
\text { ou mais em seu local de trabalho. }\end{array}$ & $\begin{array}{l}\text { Não se encontrou nenhuma associação entre } \\
\text { disfunção tireoidiana subclínica e fatores de risco } \\
\text { para doença cardiovascular. } \\
\text { Não foi verificado associação entre disfunção } \\
\text { tireoidiana subclínica e fatores psicossociais } \\
\text { (qualidade de vida, sintomas somáticos e } \\
\text { psicológicos). }\end{array}$ \\
\hline Castro $^{(35)}$ & 2012 & $\begin{array}{l}\text { Dissertação } \\
\text { Pesquisa } \\
\text { quantitativa }\end{array}$ & $\begin{array}{l}\text { Analisar métodos indiretos para } \\
\text { avaliação da composição corporal, } \\
\text { bem como algumas alterações desta } \\
\text { no processo de envelhecimento, e } \\
\text { realizar associações com fatores de risco } \\
\text { coronariano e mobilidade funcional em } \\
\text { mulheres sedentárias. }\end{array}$ & $\begin{array}{l}\text { Não houve relação significante entre sarcopenia } \\
\text { e fatores de risco para DCV, exceto para o IMC } \\
\text { como fator de proteção. }\end{array}$ \\
\hline Sousa ${ }^{(36)}$ & 2010 & $\begin{array}{l}\text { Dissertação } \\
\text { Pesquisa } \\
\text { quantitativa }\end{array}$ & $\begin{array}{l}\text { Verificar a resposta da pressão arterial a } \\
\text { um programa de caminhada controlado } \\
\text { por } 2 \text { modelos de supervisão. }\end{array}$ & $\begin{array}{l}\text { Resposta positiva na redução da PA e de fatores } \\
\text { de risco cardiovascular metabólicos em mulheres } \\
\text { hipertensas submetidas a um programa de exercício } \\
\text { físico com graus diferentes de supervisão. }\end{array}$ \\
\hline Scorsatto $^{(37)}$ & 2015 & $\begin{array}{l}\text { Tese } \\
\text { Pesquisa } \\
\text { quantitativa }\end{array}$ & $\begin{array}{l}\text { Avaliar o efeito da dieta hipoenergética } \\
\text { associada ao consumo de farinha de } \\
\text { berinjela (Solanum melongena L.) no } \\
\text { perfil lipídico e marcadores do estresse } \\
\text { oxidativo em indivíduos com sobrepeso } \\
\text { e obesidade. }\end{array}$ & $\begin{array}{l}\text { A farinha de berinjela (FB) apresentou alto teor de } \\
\text { fibras, bom conteúdo de manganês, zinco e cobre, } \\
\text { além de compostos fenólicos e saponinas com } \\
\text { importante capacidade antioxidante nos ensaios } \\
\text { in vitro. } \\
\text { A dieta hipoenergética melhorou o perfil lipídico } \\
\text { e os índices aterogênicos (triglicerídeos, HDL, } \\
\text { índice de adiposidade visceral). } \\
\text { A FB melhorou o estado antioxidante em mulheres } \\
\text { com excesso de peso, por meio do aumento da } \\
\text { capacidade antioxidante. } \\
\text { Nas mulheres obesas metabolicamente saudáveis } \\
\text { a FB aumentou a capacidade antioxidante e nas } \\
\text { mulheres obesas metabolicamente não saudáveis } \\
\text { a FB reduziu a massa gorda. }\end{array}$ \\
\hline
\end{tabular}




\begin{tabular}{|c|c|c|c|c|}
\hline Costa $^{(38)}$ & 2009 & $\begin{array}{c}\text { Tese } \\
\text { Pesquisa } \\
\text { quantitativa }\end{array}$ & $\begin{array}{l}\text { Avaliar o efeito do consumo da proteína } \\
\text { de soja isolada sobre os níveis de lipídios } \\
\text { séricos em mulheres adultas. }\end{array}$ & $\begin{array}{l}\text { Efeitos positivos com relação à redução de } \\
\text { fatores de risco para doenças cardiovasculares, } \\
\text { em especial, sobre os níveis séricos de Colesterol } \\
\text { total, LDL, após quatro semana de intervenção. } \\
\text { O consumo de proteína de soja isolada é mais } \\
\text { efetivo do que a proteína total do leite. }\end{array}$ \\
\hline Nogueira $^{(39)}$ & 2013 & $\begin{array}{c}\text { Tese } \\
\text { Pesquisa } \\
\text { quantitativa }\end{array}$ & $\begin{array}{l}\text { Avaliar em mulheres pré-hipertensas } \\
\text { obesas o efeito do consumo de chá } \\
\text { verde sobre: a pressão arterial, a } \\
\text { função endotelial, o perfil metabólico, } \\
\text { a atividade inflamatória e a adiposidade } \\
\text { corporal. }\end{array}$ & $\begin{array}{l}\text { O chá verde tem efeito benéfico sobre a pressão } \\
\text { arterial e possivelmente sobre a função endotelial. }\end{array}$ \\
\hline
\end{tabular}

$\mathrm{HAS}=$ hipertensão arterial sistêmica; $\mathrm{IMC}=$ índice de massa corpórea; $\mathrm{DM} 2=$ diabetes mellitus tipo $2 ; \mathrm{TF}=$ treinamento de força; $\mathrm{IL}=$ interleucinas; $\mathrm{PA}=$ pressão arterial; $\mathrm{PE}=$ pré eclampsia; $\mathrm{EMIC}=$ espessura médio-intimal de carótidas; $\mathrm{SM}=$ síndrome metabólica; $\mathrm{RCEst=}$ relação cintura-estatura; $\mathrm{FRCV}=$ fatores de risco cardiovascular; $\mathrm{SOP}=$ mulheres com síndrome dos ovários policísticos

\section{DISCUSSÃO}

Os fatores de risco identificados na presente revisão são, em sua maioria, modificáveis, por envolverem aspectos comportamentais e ambientais, o que indica que podem ser controlados, ou, até mesmo, prevenidos ${ }^{(5)}$. As dislipidemias são caracterizadas pelo aumento dos níveis séricos de colesterol total (CT) e do LDL, e diminuição do HDL ${ }^{(40)}$. Estudo com mulheres com câncer de mama evidenciou a importância do monitoramento constante com exames bioquímicos para identificar alterações durante o tratamento. Essas transformações, quando presentes, podem estar relacionadas a alterações do tumor, além do desenvolvimento de outras doenças, como as $\mathrm{DCV}^{(41)}$.

No que diz respeito à HAS, esta é uma condição clínica multifatorial e caracteriza-se pela elevação dos níveis de pressão arterial, associando-se a alterações estruturais e/ou funcionais dos órgãos-alvo (vasos sanguíneos, coração, rins e encéfalo), modificações metabólicas e, consequentemente, em aumento do risco de eventos cardiovasculares fatais e não fatais ${ }^{(42)}$.

O MS aponta a obesidade e o sobrepeso como fatores de risco importantes, pois apresentam efeito nocivo à saúde mesmo quando identificados de forma isolada, além de estarem relacionados ao desenvolvimento de doenças cardíacas. Ações de prevenção e diagnóstico precoce são fundamentais para a redução da morbidade e a promoção da saúde ${ }^{(43)}$. Associado à obesidade, destaca-se o sedentarismo, visto que a atividade física integra uma das principais condutas para prevenção e controle das DCV, além de contribuir na qualidade de vida da população ${ }^{(5)}$.

Quanto aos fatores de risco relacionados com os aspectos hormonais e os ciclos de vida da mulher, estudo evidencia que as mulheres que tiveram PE na gestação apresentaram frequência elevada de HAS, dislipidemia, sobrepeso/obesidade e síndrome metabólica. Reconhece-se que as alterações decorrentes da PE podem ser revertidas, após o término da gestação. Contudo, existem indicações de que muitas alterações funcionais e metabólicas podem permanecer e constituir fatores de risco para as DCV ${ }^{(44)}$.

Um alto quantitativo de pesquisas relacionou o desenvolvimento da SOP em mulherescom a presença de outros marcadores de risco cardiovascular. Esse achado associa essa síndrome a diversos fatores de risco cardiovasculares ${ }^{(45)}$. Nesse sentido, destacamse as manifestações da síndrome metabólica (SM), que podem ser observadas em muitas pacientes com SOP, envolvendo a hipertensão arterial, obesidade, tolerância diminuída à glicose e dislipidemia ${ }^{(46)}$.

Outro fator de risco relacionado ao aspecto hormonal abrangeu o período da menopausa e pós-menopausa. Conforme a Sociedade Brasileira de Cardiologia, nessa fase da vida, a mulher está mais suscetível a outros fatores, como obesidade, dislipidemia e síndrome metabólica, os quais podem acarretar em alto risco cardiovascular ${ }^{(47)}$.

É imprescindível investir em ações de promoção e prevenção, que permitam informar os indivíduos sobre os fatores de risco, para que possam ser sensibilizados e incentivados para as mudanças comportamentais quando necessárias. Essas ações podem contribuir na qualidade de vida, além de prevenir e controlar as $\mathrm{DCV}^{(48)}$.

Os resultados da presente revisão coadunam-se com a I Diretriz Brasileira de Prevenção Cardiovascular, elaborada pela Sociedade Brasileira de Cardiologia (SBC), a qual trata da magnitude das DCV no Brasil e no mundo e da importância em atuar na sua prevenção, além de enfatizar os principais fatores de risco presentes na população ${ }^{(5)}$. Evidenciou-se a ausência de pesquisas em relação a alguns fatores de risco abordados pela SBC, como o tabagismo, a diabetes mellitus, o estresse e o uso do anticoncepcional. Este é um achado relevante, pois aponta possibilidades de novas pesquisas, bem como caminhos de investigação que podem ser trilhados pela Enfermagem. Reforça-se que esses são fatores modificáveis e/ou controláveis, que podem implicar em ações de educação em saúde. 
Ressalta-se que algumas produções estavam com os resumos incompletos, o que dificultou a análise, podendo ser considerado uma limitação deste estudo. Em contraponto, a revisão permitiu reconhecer as produções sobre a temática, contribuindo para a construção do conhecimento em saúde e Enfermagem, com foco na promoção da saúde.

\section{CONCLUSÃO}

Os fatores de risco prevalentes em mulheres estão relacionados às alterações metabólicas, condições multifatoriais, hábitos inadequados, ciclos de vida, como a gestação e os aspectos hormonais, sugerindo a necessidade dos profissionais da saúde desenvolveram ações de prevenção e controle desses fatores.

\section{REFERÊNCIAS}

1. Ribeiro AG, Cotta RMM, Ribeiro SMR. A promoção da saúde e a prevenção integrada dos fatores de risco para doenças cardiovasculares. Ciênc Saúde Colet [Internet]. 2012 [acesso em 2017 Maio 17];17(1):7-17. Disponível em: http://www. nutricaoemfoco.com.br/NetManager/documentos/a_promocao_da_saude_e_a_prevencao_integrada_dos_fatores_de_risco_ para_doencas_cardiovasculares.pdf

2. Malta DC, Moura L, Prado RR, Escalante JC, Schmidt MI, Duncan BB. Mortalidade por doenças crônicas não transmissíveis no Brasil e suas regiões, 2000 a 2011. In: Ministério da Saúde (BR), Secretaria de Vigilância em Saúde. Vigitel Brasil 2014: vigilância de fatores de risco e proteção para doenças crônicas por inquérito telefônico. Brasília: Ministério da Saúde; 2015. p. 152.

3. Carlucc SEM, Gouvêa JAG, Oliveira AP, Silva JD, Cassiano ACM, Bennemann RM. Obesidade e sedentarismo: fatores de risco para doença cardiovascular. Comun Ciênc Saúde [Internet]. 2013 [acesso em 2017 Mai 17];24(4):375-84. http:// bvsms.saude.gov.br/bvs/artigos/ccs/obesidade_sedentarismo_fatores_risco_cardiovascular.pdf

4. Ministério da Saúde (BR). Vigilância das doenças crônicas não transmissíveis. Brasília: Ministério da Saúde; 2014. Disponível em: http://bvsms.saude.gov.br/bvs/publicacoes/plano_acoes_enfrent_dent_2011.pdf

5. Sociedade Brasileira de Cardiologia. I Diretriz Brasileira de Prevenção Cardiovascular. Arq Bras Cardiol [Internet]. 2013 [acesso em 2017 Maio 18];101(6):2. Disponível em: http://publicacoes.cardiol.br/consenso/2013/Diretriz_Prevencao_ Cardiovascular.pdf

6. Lelis CT, Teixeira KMD, Silva NM. A inserção feminina no mercado de trabalho e suas implicações para os hábitos alimentares da mulher e de sua família. Saúde Debate [Internet]. 2012 [acesso em 2017 Maio 18];36(95):523-32. Disponível em: http:// www.redalyc.org/pdf/4063/406341761004.pdf

7. Assis LS, Stipp MAC, Leite JL, Cunha NM. A atenção da enfermeira à saúde cardiovascular de mulheres hipertensas. Esc Anna Nery Rev Enferm [Internet] 2009 [acesso em 2017 Maio 18];13(2):265-70. Disponível em: http://www.scielo.br/pdf/ ean/v13n2/v13n2a05.pdf

8. Khader YS, Rice J, Abueita O. Oral contraceptives use and the risk of myocardial infarction: a meta-analysis. Contraception [Internet]. 2003[acesso em 2017 Maio 18];68(1):11-7. Disponível em: http://www.contraceptionjournal.org/article/S00107824(03)00073-8/fulltext

9. Schmidt MI, Duncan BB, Silva GA, Menezes AM, Monteiro CA, Barreto SM, et al. Doenças crônicas não transmissíveis no Brasil: carga e desafios atuais. Lancet [Internet]. 2011 [acesso em 2017 Maio 18]. Disponível em: http://dms.ufpel.edu. br/ares/bitstream/handle/123456789/222/1\%20\%202011\%20Doen\%C3\%A7as\%20cr\%C3\%B4nicas\%20n\%C3\%A3o\%20 transmiss\%C3\%ADveis\%20no\%20Brasil.pdf?sequence $=1$

10. Dubow C, Borba TT, Santos CR, Garcia EL, Krug SBF. Participação social na implementação das políticas públicas de saúde: uma revisão crítico reflexiva. Saúde Transform Soc [Internet]. 2017 [acesso em 2017 Maio 18];8(2):103-11. Disponível em: http://incubadora.periodicos.ufsc.br/index.php/saudeetransformacao/article/viewFile/3711/4989

11. Brasil. Lei de Direitos Autorais (Lei 9.610/98) [acesso em 2017 Jun 10]. Disponível em: http:/www.planalto.gov.br/ccivel_03/ leis/1910.htm

12. Conselho Nacional de Desenvolvimento Científico e Tecnológico. Plataforma Lattes - CNPq. Diretório dos Grupos de Pesquisa no Brasil [acesso em 2017 Jun 12]. Disponível em: http://lattes.cnpq.br/web/dgp/home

13. Ministério da Saúde (BR), Secretaria de Atenção à Saúde, Departamento de Atenção Básica. Rastreamento. Brasília: Ministério da Saúde; 2010.

14. Mansur AP, Favarato D. Mortalidade por doenças cardiovasculares no brasil e na região metropolitana de São Paulo: 
Atualização 2011. Arq Bras Cardiol [Internet]. 2012 [acesso em 2017 Jun 15];99(2):755-61. Disponível em: http://www. scielo.br/pdf/abc/v99n2/aop05812.pdf

15. Turato ER. Métodos qualitativos e quantitativos na área da saúde: definições, diferenças e seus objetos de pesquisa. Rev Saúde Pública [Internet]. 2005 [acesso em 2017 Jun 15];39(3):507-14. Disponível em: http://www.scielo.br/pdf/rsp/v39n3/24808. pdf

16. Rodrigues NLA, Lima LHO, Carvalho ES, Vera PVS, Frota KMG, Lopes MVO. et al. Fatores de risco para doenças cardiovasculares em adolescentes. Invest Educ Enferm [Internet]. 2015 [acesso em 2017 Jun 15];33(2):315-24. Disponível em: http://www.scielo.org.co/pdf/iee/v33n2/v33n2a14.pdf

17. Jose JPS. Associação entre o perfil lipídico e fatores de risco cardiovascular em mulheres de Sinop-MT [dissertação]. Goiânia: Universidade Federal de Mato Grosso; 2016.

18. Cerqueira MS. Análise de métodos antropométricos na determinação da obesidade e fatores de risco cardiovascular em mulher [dissertação]. Viçosa: Universidade Federal de Viçosa; 2011.

19. Queiroz GGJ. Efeitos da terapia interdisciplinar no controle dos fatores de risco cardiovascular e marcadores inflamatórios em mulheres obesas [dissertação]. São Paulo: Universidade Federal de São Paulo; 2013.

20. Bento CT. Associação do estado nutricional de vitamina a com índice de massa corporal, adiposidade corporal, estresse oxidativo e fatores de risco cardiovascular em mulheres com ingestão dietética recomendada de vitamina A [tese]. Rio de Janeiro: Universidade Federal do Rio de Janeiro; 2014.

21. Tibana RA. Efeitos agudos e crônicos do treinamento de força sobre fatores de risco cardiovascular em mulheres de meia idade portadoras de sobrepeso/obesidade e/ou síndrome metabólica [dissertação]. Brasília: Universidade Católica de Brasília; 2013.

22. Chagas EFB. Efeito do exercício físico de intensidade moderada sobre fatores de risco cardiovascular e marcadores inflamatórios de mulheres obesas no período pós-menpausa [dissertação]. São Paulo: Universidade Estadual Paulista Júlio de Mesquita Filho; 2013.

23. Rockett FC. Migrânea e risco para doenças cardiovasculares em mulheres [dissertação]. Porto Alegre: Universidade Federal do Rio Grande do Sul; 2013.

24. Ferreira RM. Fatores de risco cardiovascular em mulheres com antecedente de pré-eclâmpsia e sua associação com hipertrofia miocárdica e espessamento médio intimal de carótidas [tese]. São Paulo: Universidade Estadual Paulista Júlio de Mesquita Filho; 2016.

25. Andrade ACA. Sindrome metabólica e fatores associados: estudo em mulheres com pré-eclâmpsia e gestação normotensa havia cinco anos [tese] Natal: Universidade Federal do Rio Grande do Norte; 2013.

26. Costa EC. Acurácia dos índices antropométricos de obesidade central na determinação de síndrome metabólica e fatores de risco cardiovascular em mulheres com síndrome dos ovários policísticos [dissertação]. Natal: Universidade Federal do Rio Grande do Norte; 2009.

27. Azevedo MF. Níveis pressóricos elevados em mulheres com síndrome dos ovários policísticos: prevalência e fatores de risco associados [dissertação]. Natal: Universidade Federal do Rio Grande do Norte; 2010.

28. Soares EMM. Avaliação da prevalência de Síndrome Metabólica, Microalbuminúria e Risco Cardiovascular em mulheres com Síndrome dos Ovários Policísticos [Tese]. Natal: Universidade Federal do Rio Grande do Norte; 2007.

29. Soares GM. Marcadores precoces de doença cardiovascular em mulheres com síndrome dos ovários policísticos [dissertação]. São Paulo: Universidade de São Paulo; 2008.

30. Santos AGP. Risco de Framingham e evento cardiovascular em mulheres com Síndrome dos Ovários Policísticos [tese]. São Paulo: Universidade Estadual Paulista; 2014.

31. Colpani V. Fatores de risco cardiovascular em uma coorte de mulheres na menopausa no Sul do Brasil [tese]. Porto Alegre: Universidade Federal do Rio Grande do Sul; 2015.

32. Carvalho CNM. Associação entre consumo alimentar e atividade física com a síndrome metabólica em mulheres na pósmenopausa [dissertação]. Natal: Universidade Federal do Rio Grande do Norte; 2013.

33. Sousa EP. Risco cardiovascular em mulheres com câncer de mama [dissertação]. Goiânia: Universidade Federal de Goiás; 2013. 
34. Olmos RD. Doenças tireoidianas subclínicas e fatores de risco cardiovascular em mulheres com mais de 40 anos em seu local de trabalho [Tese]. São Paulo: Universidade de São Paulo; 2007.

35. Castro EA. Análise de métodos indiretos para avaliação da composição corporal, da prevalência de sarcopenia e fatores de risco cardiovascular e da relação entre osteopenia e mobilidade funcional de membros inferiores em mulheres não sedentárias [dissertação]. Viçosa: Universidade Federal de Viçosa; 2012.

36. Sousa GV. Efeito de exercício físico com modelos diferentes de supervisão sobre Hipertensão Arterial e fatores de risco cardiovascular em mulheres, numa Unidade do Programa de Saúde da Família [dissertação]. Salvador: Escola Bahiana de Medicina e Saúde Pública; 2010.

37. Scorsatto M. Efeito da dieta hipoenergética associada ao consumo de farinha de berinjela no perfil lipídico e estado antioxidante em mulheres com excesso de peso [tese]. Rio de Janeiro: Universidade Federal do Rio de Janeiro; 2015.

38. Costa CC. Efeitos do consumo de proteína de soja isolada sobre os níveis de lipídios séricos em mulheres [tese]. Rio de Janeiro: Universidade Federal do Rio Grande do Sul; 2009.

39. Nogueira LP. Avaliação do efeito do chá verde sobre a pressão arterial, função endotelial, perfil metabólico, atividade inflamatória e adiposidade corporal em mulheres pré-hipertensas obesas [tese]. Rio de Janeiro: Universidade do Estado do Rio de Janeiro; 2013.

40. Nascimento TBR, Glaner MF, Nóbrega OT. Influência do gene da apolipoproteína-E sobre a relação perfil lipídico, atividade física e gordura corporal. Rev Bras Cineantropom Desempenho Hum [Internet]. 2012 [acesso em 2017 Jun 20];14(2):22131. Disponível em: http://repositorio.unb.br/bitstream/10482/11738/1/ARTIGO_InfluenciaGeneApolipoproteina.PDF

41. Martin JL, Melnichouk O, Huszti E, Connelly PW, Greenberg CV, Minkin S, et al. Serum lipids, lipoproteins, and risk of breast cancer: a nested case-control study using multiple time points. J Natl Cancer Inst [Internet]. 2015 [acesso em 2017 Jun 20];107(5):1-9. Disponível em: https://www.ncbi.nlm.nih.gov/pmc/articles/PMC4822522/

42. Sociedade Brasileira De Cardiologia. VI Diretrizes Brasileira de Hipertensão. Arq Bras Cardiol [Internet]. 2010 [acesso em 2017 Jun 20];95(1):1-51. Disponível em: http://publicacoes.cardiol.br/consenso/2010/Diretriz_hipertensao_associados.pdf

43. Ministério da Saúde (BR). Obesidade. Brasília: Ministério da Saúde; 2006. (Cadernos de Atenção Básica, nº 12).

44. Wikström AK, Haglund B, Olovsson M, Lindeberg SN. The risk of maternal ischaemic heart disease after gestational hypertensive disease. BJOG [Internet]. 2005 [acesso em 2017 Jun 21];112(11):1486-91. Disponível em: http://onlinelibrary. wiley.com/doi/10.1111/j.1471-0528.2005.00733.x/epdf

45. Ávila MAP, Bruno RV, Barbosa FC, Andrade FC, Silva ACO, Nardi AE. Síndrome dos ovários policísticos: implicações da disfunção metabólica. Rev Col Bras Cir [Internet]. 2014 [acesso em 2017 Jun 21];41(2):106-11. Disponível em: http:// www.scielo.br/pdf/rcbc/v41n2/pt_0100-6991-rcbc-41-02-00106.pdf

46. Meirelles RMR. Menopausa e síndrome metabólica. Arq Bras Endocrinol Metab [Internet]. 2014 [acesso em 2017 Jun 21];58(2):91-6. Disponível em: http://www.scielo.br/pdf/abem/v58n2/0004-2730-abem-58-2-0091.pdf

47. Sociedade Brasileira De Cardiologia. I Diretriz Brasileira sobre Prevenção de Doenças Cardiovasculares em Mulheres Climatéricas e a Influência da Terapia de Reposição Hormonal (TRH) da Sociedade Brasileira de Cardiologia (SBC) e da Associação Brasileira do Climatério (SOBRAC). Arq Bras Cardiol [Internet]. 2008 [acesso em 2017 Jun 21]; 91(1 Supl 1):1-23. Disponível em: http://publicacoes.cardiol.br/consenso/2008/diretriz_DCV_mulheres.pdf

48. Gomes EB, Moreira TMM, Pereira HCV, Sales IB, Lima FET, Freitas CHA, et al. Fatores de risco cardiovascular em adultos jovens de um município do nordeste brasileiro. Rev bras enferm [Internet]. 2012 [acesso em 2017 Jun 21];65(4):594-600. Disponível em: http://www.redalyc.org/html/2670/267024790007/

\section{Endereço para correspondência:}

Gabriela Oliveira

Universidade Federal de Santa Maria - UFSM

Avenida Roraima, Campus Universitário, prédio 26

Bairro: Camobi

CEP: 97010-100 - Santa Maria - RS - Brasil

E-mail: gabioliveirafv@hotmail.com 